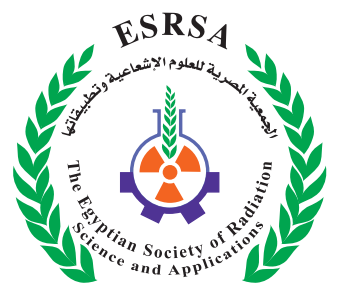

J. Nucl. Tech. Appl. Sci., Vol. 9, PP. 13 : 18 (2021)

\title{
Yeast Cells Handling Using Reproducible Ultrasound Standing Wave Chamber \\ Design: An In Vitro Approach
}

Moustafa, M.H.

Received: 16/06/2020

Accepted: 23/02/2021

DOI: $10.21608 /$ jntas.2021.43960.1029

E.mail:moustafa-hm@alexu.edu.eg

\section{ABSTRACT}

Handling and manipulation of biological cells are fundamental for biotechnology applications like genes modification, membrane fusion, and drug researches. Cells in suspension can be handled by external applied force fields. These force fields can be immunological, mainly depends on membrane binding criteria of living cells, or non-immunological, utilizing physical properties such as cell size and shape. Hereby, dry bakers' yeast cells suspension were handled by the aid of a simple homemade ultrasonic standing wave chamber operating at a frequency of $0.8 \mathrm{MHz}$, with power intensities ranging $0.5-3 \mathrm{~W} / \mathrm{cm}^{2}$ in pulsed mode. Microscopic monitoring revealed cells trapping in nearly ultrasonic wave's interference nodes by the acoustic radiation forces. The acoustic effect on trapping time and velocity of the cell were investigated. The trapping be-

\section{KEYWORDS}

Cell Trapping,

Ultrasounds, Standing Waves, Radiation Forces, Biotechnology. havior depends mainly on the cell size, and ultrasounds power intensity. Increasing the ultrasounds intensity reduces the time consumed to trap cells. In summary, acoustic forces generated in standing wave mode may be a simple promising noninvasive tool for biological cell handling in many biotechnology applications.

1. Biomedical physics Department, Medical research institute, Alexandria University, Alexandria, Egypt. 


\section{INTRODUCTION}

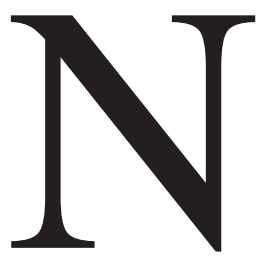

on-invasive, contactless handling tools for bio-particles (e.g. cells, viruses, and functionalized beads) have crucial application in biotechnology. Cell separation can be carried out by immuno-reactions of membrane protein with the capturing antibodies. High specificity and selectivity are the main advantages of these tools. On the other the hand, the high cost and complicated processing remain as challenges (Saito et al., 2002).

Meantime, non-immunological methods are relatively fast and simple techniques. In these methods, the cells are determined and separated according to their physical properties such as, cell size and shape. Thus, a non-immunological technique is expected to be a promising separation method. These tools either have high spatial accuracy (e.g. dielectrophoresis DEP, and laser tweezers for slow 3D handling of individual particle) or long range (e.g. ultrasonic standing waves) for high-throughput of large particle ensembles (Taha et al., 2017 and Saito et al., 2002).

In spite of good results obtained by using DEP and optical methods, they still have some common disadvantages, as follows: in electrical means, the demand of integrated micro-structured electrodes with stable manipulation will increase the costs for system fabrication; specific electric properties are required for the samples; the electromagnetic fields disrupt the properties of the cells, which is not desirable for instance when the kinetics of particles (such as cells or other biological material) is studied in an unperturbed fluid environment (Mizutani et al., 2019 and Dovinikov et al., 2020).

Acoustofluidics are other handling tools that deal with acoustics and exert radiation force which can be used for gentle handling of the cells and other particles within the field. Particles subjected to acoustic waves are influenced by acoustic radiation forces, which are particularly strong in standing wave fields
(GrÖschl et al., 1996 and Taha et al., 2017).

Ultrasound Standing Wave (USW) manipulation technique has been successfully used to manipulate and separate biological particles in macro- and micro-scale devices and it became widely spread by the mid-eighteenths (Di Giacinto et al., 2019 and GrÖschl et al., 1996). This technique was effectively used for concentration of mammalian cells (blood cells, stem cells, cells of various tissues), ultra-phytoplankton and, bacteria, etc. Beside, it has no effect on survival and integrity of these manipulated biological cells (Ostasevicius et al., 2018 and Moradi et al., 2020)

The first idea to use ultrasounds was to coagulate dust and smoke. Meanwhile, the acoustic manipulation ideas to manipulate particles with ultrasound have been clarified in late nineteenth (Kremkau $\boldsymbol{e t}$ al., 1998). An efficient piezoelectric resonator for particle separation emphasizing on the study of their geometry, dimensions and material was constructed by (Gröschl et al., 1996). Further modifications were made to enhance performance for more dedicated applications involving gene therapy and transduction (Mizutani et al., 2019 and Dovinikov et al., 2020).

Herein, the present work aims at establishing a home-made therapeutic ultrasound standing wave chamber for baker's yeast cells. An in vitro approach was used to predict operating conditions.

\section{MATERIALS AND METHODS}

\section{Yeast cell preparation}

Yeast cells are well studied, simple unicellular cells with unique features for ease of growth and genetic manipulation, making them an exceptional host for microbes and good candidates for medical applications (Roohvand $\boldsymbol{e t}$ al., 2017). For the present study, the bakery dry yeast cells were purchased from local markets. Two grams yeast powder was added to $10 \mathrm{ml}$ distilled water pretreated with traces 
of table sugar (to maintain yeast cell viability) with concomitant stirring at $37^{\circ} \mathrm{C}$. The cells concentration was counted using a Neubauer haemocytometer to yield a final suspension of about $1.5 \times 10^{5}$ cells/ ml.

\section{Ultrasonic source}

The ultrasonic generator (Model CSL Shanghai, No822 Factory, China) operating at $0.8 \mathrm{MHz}$ con- tinuous wave mode, with an output intensity ranging from 0.5 to $3 \mathrm{~W} / \mathrm{cm}^{2}$, was used as a source for the ultrasonic waves. This instrument uses alternating current electronic tube to generate oscillation by a calcium zirconate titanate circular transducer. The emitted ultrasonic (US) waves is coupled to the trapping chamber using an aluminum sheet extended inside the chamber as shown in Figure 1.

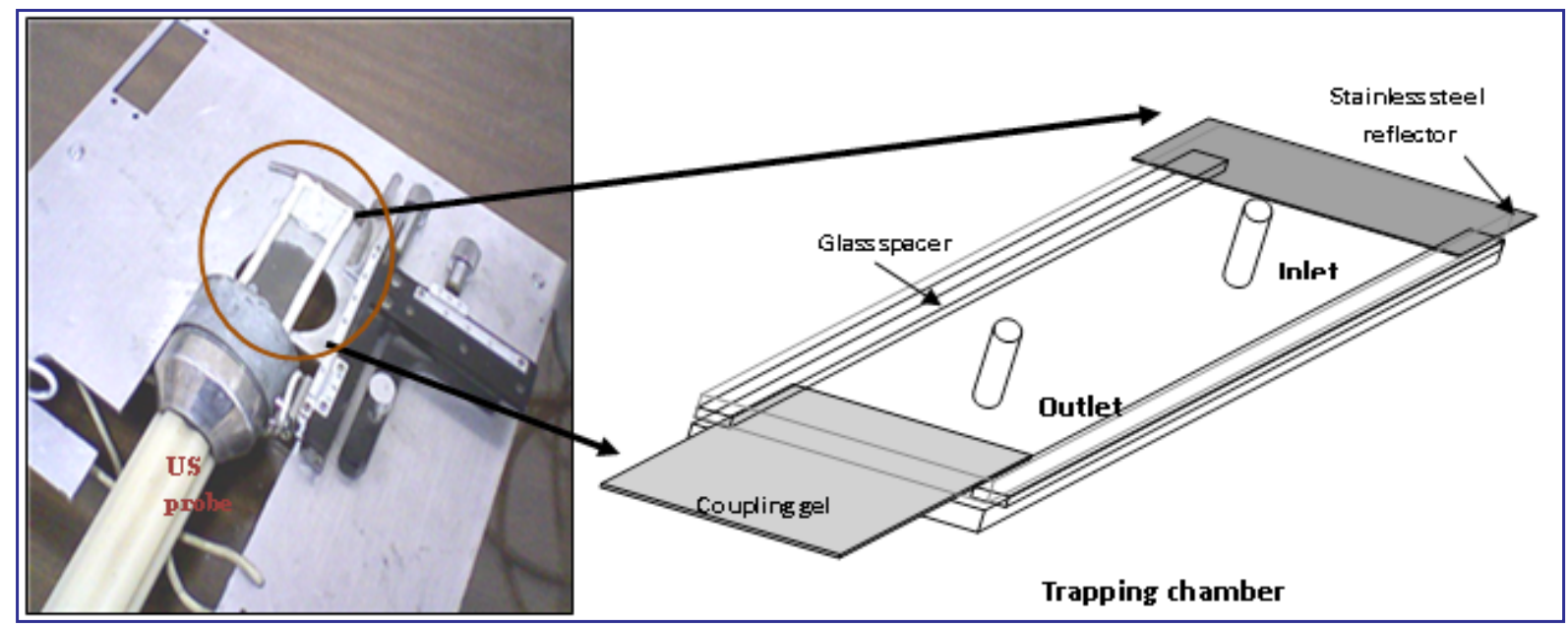

Fig. (1): the basic set-up of cell handling ultrasonic standing wave chamber (left side) with diagrammatic scheme for the chamber components (right side).

Studying the effect of Ultrasound intensity on trapping efficiency Cell trapping fraction

To evaluate the yeast cells trapping efficiency, trapping fraction was measured with US intensity. Trapping fraction was calculated. Trapping fraction is defined as the percentage of the trapped cells after a definite time, as in equation 1. Captured photos of (400 x) microscopic magnification were filtered using Scion image program (version 4.0.3.2) to calculate the cell trapping fraction. Time required to trap all cells and form a trapping band was recorded using a stopwatch.

Trapping.. fraction $(\%)=$ $\frac{\text { mean no. of trapped cells/imag e }}{\text { mean total no. of cells/imag e }} \times 100$ Equation 1

\section{The effect on cell Trapping Velocity}

From the recorded film of the cell during trap- ping, the instant cell velocity was calculated by measuring the time required for the cell to travel $10 \mathrm{~mm}$ on the PC screen along the trapping path 15 seconds after the ultrasounds application. The cell movement distance on the screen was calibrated using standard latex sphere particles of diameter equal to $1.151 \mu \mathrm{m}$. the velocity of the cell was estimated from the relation given below:

Cell. velocity $=\frac{\text { Distance }(\mathrm{mm})}{\text { Time consumed (seconds) }}$ Equation 2

\section{RESULTS}

Figure 2 shows the light microscopic image of the cells patterns before and after sonication with $0.8 \mathrm{MHz}$ ultrasonic waves operated at intensity of 3 $\mathrm{W} / \mathrm{cm}^{2}$.Before sonication (left side image), the cells were distributed randomly and after sonication (right side image), the cells begin to stratify in organized bands after nearly 30 seconds. 


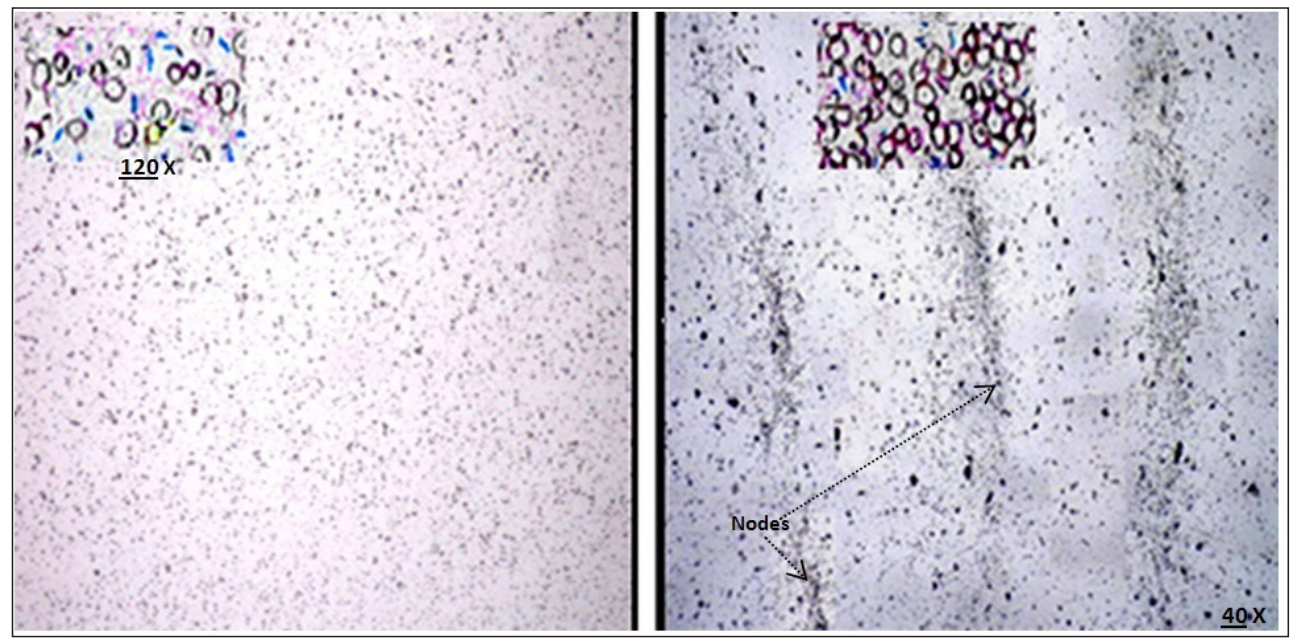

Fig. (2): Light microscope images of the yeast cells (blue rods) before $(120 \mathrm{x})$ and after $(40 \mathrm{x})$ sonication of $0.8 \mathrm{MHz}$ ultrasound waves at $3 \mathrm{~W} / \mathrm{cm} 2$ intensity.

Rate of changes in the number of yeast cells in trapping bands is shown in microscopic photographs of Figure 3, the cell are randomly distributed (a), as the time proceeds the cells behave to trap (b) till formation of visible bands (c), then at prolonged time the cells become to adhere more to each other (d).

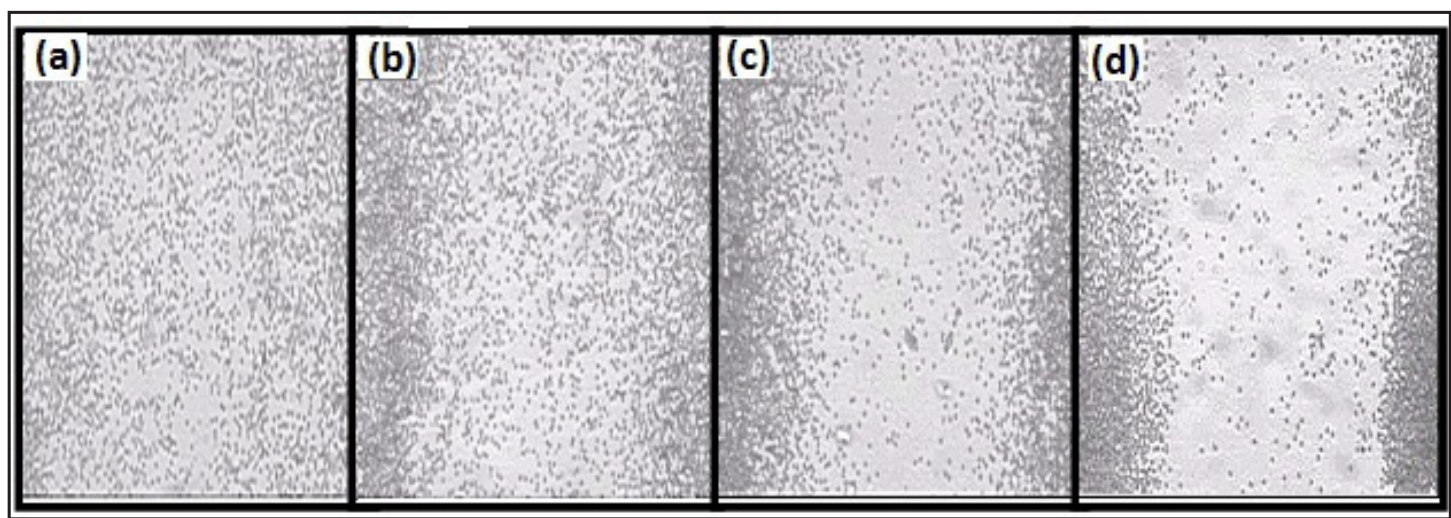

Fig. (3): Sonication time effect on cell trapping band formation.

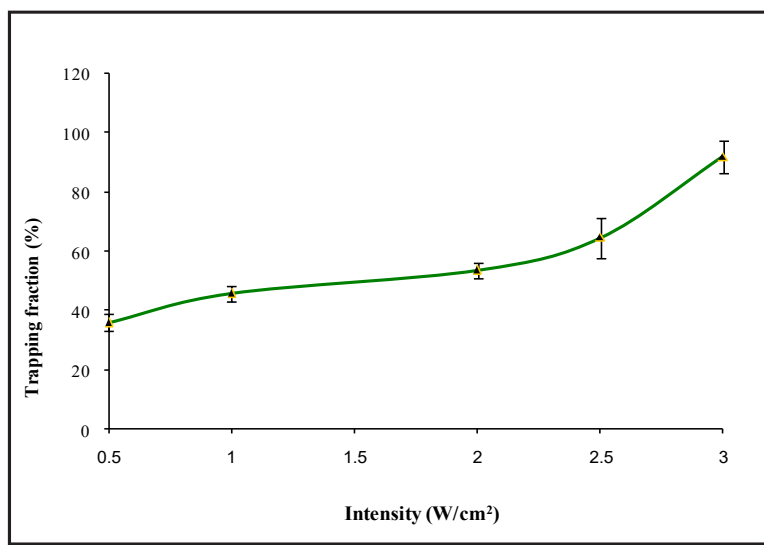

Fig. (4): the relation between yeast cell trapping fraction with US intensity (p-value $<0.05$ ).

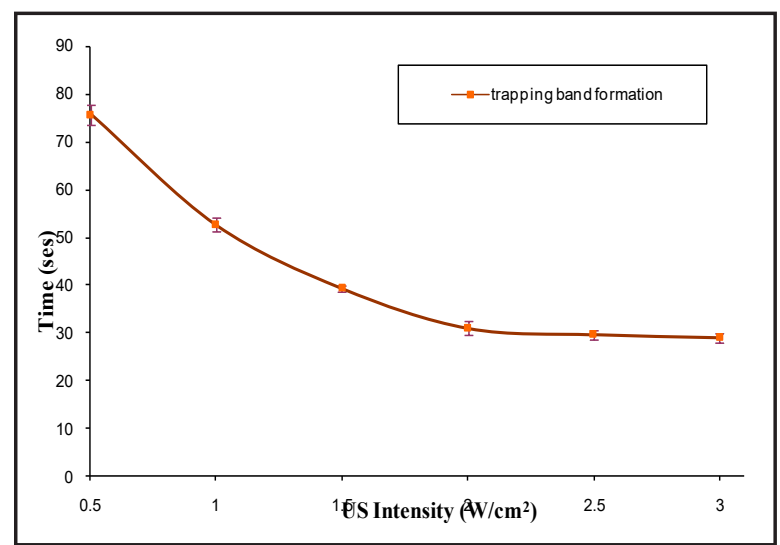

Fig. (5): the time consumed for trapping bands appearance for each corresponding US intensity value ( $p$-value $<0.05$ ). 
The effect of increasing the applied US intensity on trapping fraction is shown in Figure 4 . It was found that the increasing the applied US intensity significantly raises the percentage of trapping fraction per image studied, and the time required for trapping as illustrated in Figure 5. That is an indication of increasing in trapping efficiency.

\section{DISCUSSION}

In ultrasound standing wave, irradiated cells are influenced by two main types of forces; primary and secondary. These forces mainly originate from the standing wave itself and the due to sound waves scattered by the cells respectively (Doinikov $\boldsymbol{e t}$ al., 2020).

Primary radiation force (PRF) can be divided into the axial component and the transverse component (equation 3, equation 4). The axial PRF acts in the direction of the acoustic wave field propagation, and is stronger than the transverse one. The axial PRF translates particles or cells to either the nodes or the antinodes of the standing wave. The transverse PRF is subsequently responsible for packing the particles closer together and to withhold their positions (Liang et al., 2019 and Doinikov et al., 2018).

$$
\begin{aligned}
& \mathrm{F}_{\mathrm{PRF}}=-\left(\frac{2 \pi \mathrm{r}^{3} \mathrm{P}}{\lambda . v}\right) \times \phi(\beta, \rho) \times \operatorname{Sin}\left(\frac{4 \pi x}{\lambda}\right) \text { Equation } 3 \\
& \phi=\frac{5 \rho_{\mathrm{c}}-2 \rho_{\mathrm{m}}}{2 \rho_{\mathrm{c}}+\rho_{\mathrm{m}}}-\frac{\beta_{\mathrm{c}}}{\beta_{\mathrm{m}}} \text { Equation } 4
\end{aligned}
$$

The axial PRF states that the acoustic force is proportional to the acoustic wave intensity $(\mathrm{P})$, and the size of the particle $\left(\mathrm{r}^{3}\right)$. As a result, as the particle diameter is reduced, the acoustic force diminishes rapidly, and also the acoustic contrast factor $(\phi)$. Particles with positive contrast factor are driven toward pressure nodes (velocity anti-node), while they are driven toward antinodes for a negative contrast factor. Studying of the trapping pattern indicate that the interspace distances between the center of the bands is nearly $0.9 \mathrm{~mm}$, which is nearly equal to half wavelength of the ultrasonic waves used (Groschl, 1996 and Wiklund et al., 2006).

A simplified equation for the inter-particle forces (equation 5), when the acoustic wavelength is much greater than the particle radius (Wang et al., 2014 and Doinikov et al., 2020) is given below:

$$
\begin{aligned}
& \mathrm{F}_{\mathrm{B}}(\mathrm{x})=4 \pi r^{6}\left[\frac{\left(\rho_{\mathrm{c}}-\rho_{\mathrm{m}}\right)^{2}\left(3 \operatorname{Cos}^{2} \theta-1\right)}{6 \rho_{\mathrm{m}} \mathrm{d}^{4}} \mathrm{v}^{2}(\mathrm{x})\right. \\
& \left.-\frac{\omega^{2} \rho_{\mathrm{m}}\left(\beta_{\mathrm{c}}-\beta_{\mathrm{m}}\right)^{2}}{9 \mathrm{~d}^{2}} \mathrm{p}^{2}(\mathrm{x})\right] \text { Equation } 5
\end{aligned}
$$

where, $(r)$ is the radius of the particle, $(d)$ is the distance between the particles, and $\square$ is the angle between the centre line of the particles and the direction of propagation of the incident acoustic wave.

The sign of the force is to be interpreted such that a negative sign means an attractive inter-particle force, and a positive sign means a repulsive force. The left side of the equation depends on the particle velocity amplitude $\mathrm{v}(\mathrm{x})$, and the right side depends on the acoustic intensity $\mathrm{p}(\mathrm{x})$.

\section{CONCLUSION}

In conclusion, the current work presented noninvasive, contactless tool using ultrasound standing wave for living cells handling and manipulation. Simple, cheap, affordable home made trapping chamber was designed. Yeast cells in suspension were influenced by acoustic radiation forces that are proportional to the acoustic wave intensity, cell size. The cell trapping was monitored by the mean of trapping fraction and trapping time with different ultrasonic wave intensities. Overall ultrasonic standing waves can be considered a reasonable non-invasive cell handling tool for gene/ drug delivery, in biotechnology applications. 


\section{REFERENCES}

- Doinikov, A.A.; Gerlt, M.S. and Dual, J. (2020): Acoustic Radiation Forces Produced by Sharp-Edge Structures in Microfluidic Systems. Phys. Rev. Lett., 124(15): 154501.

- Doinikov, A.; Thibault, P. and Marmottant, P. (2018): Acoustic streaming induced by two orthogonal ultrasound standing waves in a microfluidic channel. Ultrasonics, 87: 7.

- Liang, S. and Chaohui, W. (2018): Revised model for the radiation force exerted by standing surface acoustic waves on a rigid cylinder. Physical Review, E 97.

- Groschl, M. (1996): Ultrasonic Separation of Suspended Particles. Acustica acta acustica, 84: 432.

- Hedrick, W.; Hykes, D. and Starchman, D. (2005): Ultrasound Physics and Instrumentation. St. Louis, Mo.: Elsevier Mosby.

- Mizutani, H. and Saito, T. (2019): Innovative use of low-frequency ultrasound for particle separation/ classification: Forces acting on a single particle held in water of 20-kHz-ultrasound pressure fields in transition states under control of the acoustic pressure amplitude. Chem. Eng. Sci., 206: 476.

- Petersson, F.; Nilsson, A.; Holm, C.; Jönsson, H. and Laurell, T. (2005): Continuous separation of lipid particles from erythrocytes by means of laminar flow and acoustic standing wave forces. Lab Chip, 5(1): 20.

- Roohvand, F.; Shokri, M.; Abdollahpour-Alitappeh, M. and Ehsani, P. (2017): Biomedical applications of yeast- a patent view, part one: yeasts as workhorses for the production of therapeutics and vaccines. Expert Opin. Ther. Pat., 27(8): 929.

- Saito, M.; Kitamura, N. and Terauchi, M. (2002): Ultrasonic manipulation of locomotive microorganisms and evaluation of their activity. J. Appl. Phys., 92(12): 7581.
- Taha, T.; Soliman, A. and Eldosoky, M. (2017): Analysis improvement of standing surface acoustic wave microfluidic devices for bio-particles separation. Int. J. Comput. Appl. Technol., 55(3): 244.

- Wang, L. and Li, S. (2014): Acoustic Field Calculation of Ultrasonic Phased Array Concave Cylindrical Transducer. Appl. Mech. Mater., 716-717: 1111.

- Wiklund, M.; Günther, C.; Lemor, R.; Jäger, M.; Fuhr, G. and Hertz, H. (2006): Ultrasonic standing wave manipulation technology integrated into a dielectrophoretic chip. Lab Chip, 6(12): 1537. 